\title{
Firing Properties of Spinal Interneurons during Voluntary Movement. I. State-Dependent Regularity of Firing
}

\author{
Yifat Prut ${ }^{2}$ and Steve I. Perlmutter ${ }^{1}$ \\ ${ }^{1}$ Department of Physiology and Biophysics and the Washington National Primate Research Center, University of Washington, Seattle, Washington 98195, \\ and ${ }^{2}$ The Hebrew University, Hadassah Medical School, Jerusalem 91120, Israel
}

The firing properties of single spinal interneurons (INs) were studied in five awake, behaving monkeys performing isometric or auxotonic flexion- extension torques at the wrist. INs tended to fire tonically at rest (mean rate, 14 spikes (sp)/sec) and during generation of static torque (mean rate, $19 \mathrm{sp} / \mathrm{sec}$ in flexion, $24 \mathrm{sp} / \mathrm{sec}$ in extension). INs exhibited regular firing, with autocorrelation functions showing clear periodic features and a mean coefficient of variation of interspike intervals $(\mathrm{CV})$ of 0.55 during production of static torque. For the population, there was an inverse correlation between $\mathrm{CV}$ and mean rate. However, $46 \%$ of the INs had task-dependent changes in regularity that were not predicted by changes in firing rate, suggesting that their firing pattern is determined not only by the intrinsic properties of the neurons but also by the properties of its synaptic inputs. INs showed two main response types to passive wrist displacement: biphasic and coactivation. Cells with these sensory responses had different, stereotypical temporal activity profiles and firing regularity during active movement. However, INs having correlational linkages with forearm muscles, identified as features in spiketriggered averages of electromyographic activity, did not exhibit unique responses or firing properties, although they tended to fire more regularly than other INs. This suggests the lack of a precise mapping of inputs to outputs for the spinal premotor network.

Key words: spinal cord; interneurons; primates; firing pattern; synchronization; motor control

\section{Introduction}

Primates can rapidly execute different forelimb movements that require activation of different sets of arm muscles. Functional synergists in one behavior may act as antagonists in the next. The flexibility of spinal circuitry contributes significantly to this motor versatility, as demonstrated by the task-dependent modulation of spinal reflexes (Stein and Capaday, 1988; LaBella et al., 1992; Floeter et al., 1993; Pearson, 1995). Transmission through spinal pathways is altered by modifying synaptic inputs and neuronal excitability. These mechanisms produce behaviorally dependent changes in the input-output transformations performed by spinal interneurons (INs) and motoneurons (MNs).

We can elucidate mechanisms of muscle pattern recruitment by characterizing these transformations in behaving animals. Our previous studies related the discharges of spinal neurons to parameters of movement or muscle activation (Maier et al., 1998; Perlmutter et al., 1998; Prut and Fetz, 1999). Another approach examines the temporal firing properties of neurons and has yielded valuable results in other systems. Discharge regularity in auditory pathways is correlated with physiological and morphological properties of neurons (Young et al., 1988; Rees et al., 1997), and the temporal firing patterns of Ia afferents and spinal

Received March 21, 2003; revised July 30, 2003; accepted Aug. 19, 2003.

This work was conducted at the Washington National Primate Research Center, University of Washington, and was supported by National Institutes of Health Grants NS36781, NS12542, and RR00166, the United States-Israel Binational Science Foundation, and the Israel Science Foundation. We thank Larry Shupe and Jonathan Garlid for technical support and Moshe Abeles and Eberhard Fetz for valuable comments on this manuscript.

Correspondence should be addressed to Yifat Prut, Department of Physiology, The Hebrew University, Hadassah Medical School, P.0. Box 12272, Jerusalem 91120, Israel. E-mail: yifat@md.huji.ac.il.

Copyright $\odot 2003$ Society for Neuroscience $\quad$ 0270-6474/03/239600-11\$15.00/0 neurons are modified by changes in $\gamma$ drive or synaptic input (Matthews and Stein, 1969; Dengler et al., 1986; Wiegner et al., 1993; Butt et al., 2002).

The discharge variability of a neuron is determined by its intrinsic properties, particularly afterhyperpolarization and refractoriness (Person and Kudina, 1972; Bennett and Wilson, 1999; Piotrkiewicz, 1999; Powers and Binder, 2000), and by the temporal and spatial patterns of synaptic activation (Calvin and Stevens, 1968; Stevens and Zador, 1998; Gonzalez-Forero et al., 2001). Thus, changes in discharge variability during movement can reflect changes in the inputs of a cell and may help reveal how transmission through spinal pathways is modified during behavior.

Changes in temporal properties not only may reflect changes in transmission through neural networks but also may mediate altered transmission. It is well established that the variability of spiking in a presynaptic axon can affect the firing of the postsynaptic cell (Segundo et al., 1963). For example, transition of a cell from bursting to regular firing can change its effectiveness in driving the discharge of postsynaptic neurons. The regularity of discharge of auditory neurons may enhance the coding of some stimulus parameters (Goldberg et al., 1964; Young et al., 1988; Rees et al., 1997). Hypotheses about the function of local circuits in the cerebral cortex have assigned important roles to temporal firing characteristics (Softky and Koch, 1993; Mainen and Sejnowski, 1995; Riehle et al., 1997; Stevens and Zador, 1998; Salinas and Sejnowski, 2000). In the spinal cord, changes in the firing properties of INs could affect the ability of converging inputs to drive motoneurons.

We studied the firing properties of INs and MNs in awake, 
behaving monkeys performing voluntary wrist movements. This paper reports on single-cell properties, and the companion paper (Prut and Perlmutter, 2003) examines the extent and effects of common input to pairs of simultaneously recorded INs.

\section{Materials and Methods}

Behavioral paradigm

The methods have been described at length previously (Maier et al., 1998; Perlmutter et al., 1998; Prut and Fetz, 1999) and are briefly summarized here. Five macaque monkeys (Macaca nemestrina) were trained to perform visually guided flexion and extension of the wrist for applesauce rewards. Torque generated about the wrist controlled the position of a cursor on a video monitor. The monkeys were required to generate torque to shift the cursor into target boxes that appeared on the left or right side of the screen and to maintain the cursor within the box for a variable period of $2-3 \mathrm{sec}$, called the active-hold period. The active-ramp period was defined as the time from dynamic torque onset to attainment of the static torque level. Monkeys generated torque either isometrically or against an elastic load.

The first monkey (B) was presented with alternating flexion and extension targets (Fig. 1E). Target boxes were located at different eccentric positions, requiring the monkey to generate different torque levels. The second monkey (W) performed a similar task that included a center target box, corresponding to no torque exerted at the wrist (Fig. $1 F$ ). After positioning the cursor within the center box for 1-2 sec (rest period), the center box was extinguished, and, simultaneously, a new target appeared either to the left or to the right. The monkey generated wrist torque to move the cursor into the new target, held it there for an activehold period, and then had to reposition the cursor within a second center box before obtaining the reward. The other monkeys (E, F, and H) were trained to perform an instructed delay task (Fig. $1 G$ ), in which a visual cue informed the monkey of the location of the target 1-2 sec before a movement to that target was permitted (Prut and Fetz, 1999). For monkey $\mathrm{H}$, proprioceptive cues were used in addition to visual cues in alternating blocks of trials. Proprioceptive cues were small, passive displacements of the wrist in either flexion or extension (Fig. $1 H$ ). In this paper, we focus on rest and active-hold periods and do not consider the activity of cells during the delay period. The terminology and timing of the different epochs in each of the trials are shown in Figure $1 E-H$.

\section{Recording techniques}

After each monkey was fully trained, a device to stabilize the head was mounted on the skull. A unilateral laminectomy of the C5-T1 vertebrae was performed, and a stainless steel recording chamber was implanted over the lower cervical spine. Bipolar electromyographic (EMG) electrodes were implanted in 10-14 forearm muscles, typically extensor carpi ulnaris, extensor carpi radialis, extensor digitorum communis, extensor digitorum 2 and 3 , extensor digitorum 4 and 5, abductor pollicis longus, brachioradialis, flexor carpi radialis, flexor carpi ulnaris, flexor digitorum profundus, flexor digitorum superficialis, palmaris longus, and pronator teres. EMG recordings were made through multistranded stainless steel wires implanted transcutaneously in monkeys B, E, and F. Patch electrodes (Microprobe, Clarksburg, MD) and wires were surgically implanted in monkeys $\mathrm{W}$ and $\mathrm{H}$. Activity of spinal neurons was recorded extracellularly in segments $\mathrm{C} 6-\mathrm{T} 1$ through one or two glasscoated tungsten electrodes.

\section{Data collection}

Neuron and muscle activity, wrist torque, and task-related behavioral events were recorded on multichannel FM tape (TEAC XR7000). One or more spikes were sorted off-line from the neuron signal using either a time-amplitude window discriminator or a spike-sorting device based on a template-matching algorithm (Alpha Omega Engineering, Nazareth, Israel). The quality of isolation was continuously monitored throughout the spike-sorting process. Sorted units were further tested for the existence of an absolute refractory period of $\geq 1 \mathrm{msec}$ and a gradual decay of the left tail of the interspike interval (ISI) histogram, reflecting a relative refractory period. The stationarity of single-cell activity was checked by inspecting the average rate and the task-related rate modula- tion of each cell as a function of time. Cells that exhibited a trend in these two measures were omitted from the study.

Data were collected from 776 units from monkeys B, W, E, and F. Of these, 387 (13 MNs and 374 INs) passed all criteria and were considered to be single, well isolated, stationary neurons. Our criteria were stringent to avoid, as much as possible, the inclusion of single-unit records that were contaminated by the activity of nearby cells. This precaution is particularly important when studying the regularity of neuronal firing. In addition, data from 38 well isolated INs that were recorded from monkey $\mathrm{H}$ were analyzed separately for responses to passive displacements of the wrist.

The anatomical distance between cells recorded on different electrodes was hard to reconstruct. We therefore assumed that neurons recorded simultaneously by the same electrode were located in close proximity, and those recorded by two different electrodes were located relatively far from each other.

\section{Data analysis}

Correlational linkages between neurons and muscles. Spike-triggered averages (STAs) of rectified EMG recordings were compiled to determine the correlational linkages between recorded neurons and forearm muscles (Cheney and Fetz, 1980). Only STAs for which there were $\geq 2000$ triggers were analyzed. Three types of features were observed in STAs of EMG recordings (Perlmutter et al., 1998). Motor unit action potentials appeared as robust features in STAs of both rectified and unrectified EMG recordings, starting shortly after spike onset. Postspike effects appeared as increases or decreases in averaged muscle activity after the occurrence of the trigger spike with onset times consistent with synaptic connections between the trigger cell and MNs (for criterion, see Perlmutter et al., 1998). Synchrony effects were increases or decreases in averaged muscle activity that started before and ended after the trigger spike. Such effects cannot be explained by serial, synaptic linkages from the trigger cell to the affected muscle. Sixty-three INs had postspike effects alone; 36 INs had synchrony effects alone; and 34 had both. There were no significant differences between the temporal properties of INs with postspike effects, synchrony effects, or both, and the data were usually combined (referred to as INs with correlational linkages to muscles). Cells that exhibited no features in STAs were classified as unidentified interneurons (U-INs).

Task-modulated activity. Neurons with task-modulated activity were identified by performing paired $t$ tests of single-trial, average firing rate during the rest and movement periods across all trials. The $t$ tests were performed separately for the active-ramp and active-hold periods to determine the presence of phasic and tonic response components, respectively. The preferred direction of a neuron (if any) was determined for the active-ramp and active-hold periods by performing a $t$ test between the single-trial, average rates during flexion and extension. The significance level for all $t$ tests was taken as $5 \%$. Response profiles during the hold period (tonic, incrementing, decrementing, and no activity) were assigned subjectively.

Regularity of firing. The regularity of firing of single neurons was quantified by the coefficient of variation of interspike intervals (CV). Two measures were used in this study. The first was termed the average CV and was based on the coefficient of variation for individual trials $\left(C V_{\mathrm{tr}}\right)$, defined as:

$$
\mathrm{CV}_{\mathrm{tr}}=\frac{\sigma\left(\mathrm{ISI}_{\mathrm{tr}}\right)}{E\left(\mathrm{ISI}_{\mathrm{tr}}\right)}
$$

where $t r$ is the trial index; $I S I_{\text {tr }}$ is the set of interspike intervals in a single epoch in an individual trial; $\sigma$ is the SD; and $E$ is the mean. $\mathrm{CV}_{\mathrm{tr}}$ was computed separately for the rest and active-hold periods and only for the last second of each period so that transient responses during dynamic torque production were excluded. Only epochs with more than five interspike intervals were considered. We performed simulations of regular and Poisson-distributed point processes with a range of mean firing rates to determine the effect of using a minimum of between 5 and 10 intervals to estimate $\mathrm{CV}_{\mathrm{tr}}$. Using a larger number of minimal intervals did not systematically improve the estimate because it resulted in fewer acceptable trials. The average CV for each neuron was computed as the mean of 
$\mathrm{CV}_{\mathrm{tr}}$ for all trials and was calculated separately for the rest and activehold periods. Only neurons for which there were data from $>10$ trials were included. The coefficient of variation was not calculated for the active-ramp period.

Relationship between regularity and firing rate. The second measure of regularity was the instantaneous coefficient of variation $\left(\mathrm{CV}_{\mathrm{i}}\right)$, computed over a fixed number of intervals. For each neuron, entire epochs (rest and active-hold periods) in individual trials were divided into successive series of 10 ISIs. $\mathrm{CV}_{\mathrm{i}}$ was taken as the coefficient of variation during this 10-ISI window. Simulations of regular and Poisson-distributed point processes determined that using only 10 interspike intervals to calculate $\mathrm{CV}_{\mathrm{i}}$ gives a small underestimate for Poisson processes only, but the magnitude of this underestimate is independent of mean firing rate. $\mathrm{CV}_{\mathrm{i}}$ was plotted as a function of the mean firing rate during the 10-ISI window for all windows in all trials. To detect taskdependent relationships between regularity of firing and mean rate, the data were fit by the following exponential equation using a nonlinear, least-squares Gauss-Newton algorithm (with a maximal iteration number of 1000 and a tolerance of 0.001 ):

$$
\mathrm{CV}_{\mathrm{i}}(\text { rate })=a e^{\left(-\frac{\text { rate }}{\tau}\right)}+c .
$$

We chose this relationship because an exponential function is expected for interspike intervals of MNs (Powers and Binder, 2000) (R. K. Powers, personal communication). The residuals of the exponential fit were calculated by subtracting the fitted values from the raw data. The residuals were then tested for behaviorally related effects using a nonparametric, one-way ANOVA (Kruskal-Wallis, $p<0.05$ ) between rest and active-hold periods and between flexion and extension trials. This test was conducted only for epochs with at least five $\mathrm{CV}_{\mathrm{i}}-$ rate data points.

In some cases, the $\mathrm{CV}_{\mathrm{i}}$-rate plots suggested a linear relation, and we computed the sum of the absolute deviations of the data from exponential and linear fits to compare the goodness of fits. An exponential $\mathrm{CV}_{\mathrm{i}}$-rate function might be fit well by a linear function when data are available only over a limited range of firing rates, as was the case for some neurons in the present study.

Onset time of perturbation and movement responses. The onset times of neuronal responses to passive displacements of the wrist were measured in monkey $\mathrm{H}$. A poststimulus time histogram (PSTH, $1 \mathrm{msec}$ bins) of neuronal activity was compiled for -200 to $+500 \mathrm{msec}$ around the time of the perturbation. For cells that were active during the $200 \mathrm{msec}$ before the perturbation, the entire PSTH was convolved with a Gaussian kernel that had an SD of $4 \mathrm{msec}$ and spanned 12 bins. Response onset was defined as the time after the start of the command pulse delivered to the torque motor that the PSTH crossed the preperturbation mean $+3 \mathrm{SD}$. The timing of this pulse either coincided with or occurred one sample $(10 \mathrm{msec})$ before the beginning of wrist displacement. Onset time was defined for excitatory responses only. For neu- rons with no background activity, the PSTH was not smoothed, and onset time was taken as the occurrence of the first spike of the response.

The onset times of IN responses during active movement were calculated similarly. Histograms of unit activity with $5 \mathrm{msec}$ bins were aligned on torque onset and smoothed as above. Response onset was defined as

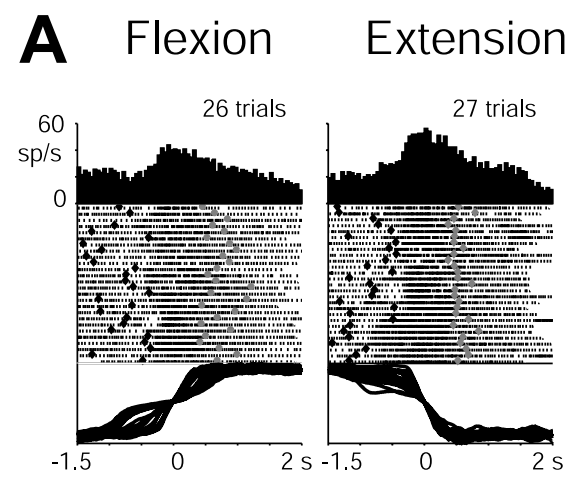

E Alternating

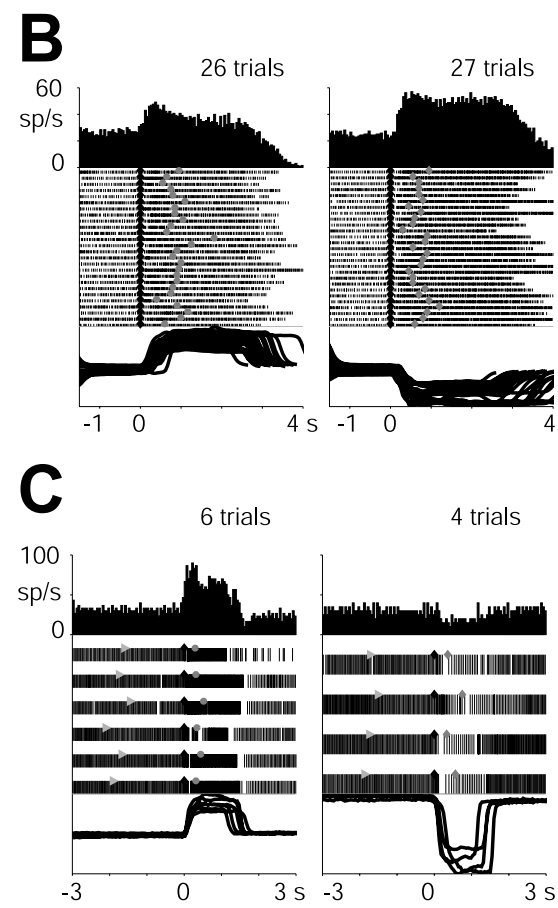

F Center-out

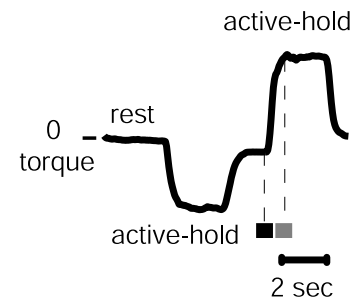

G Delay - visual cue
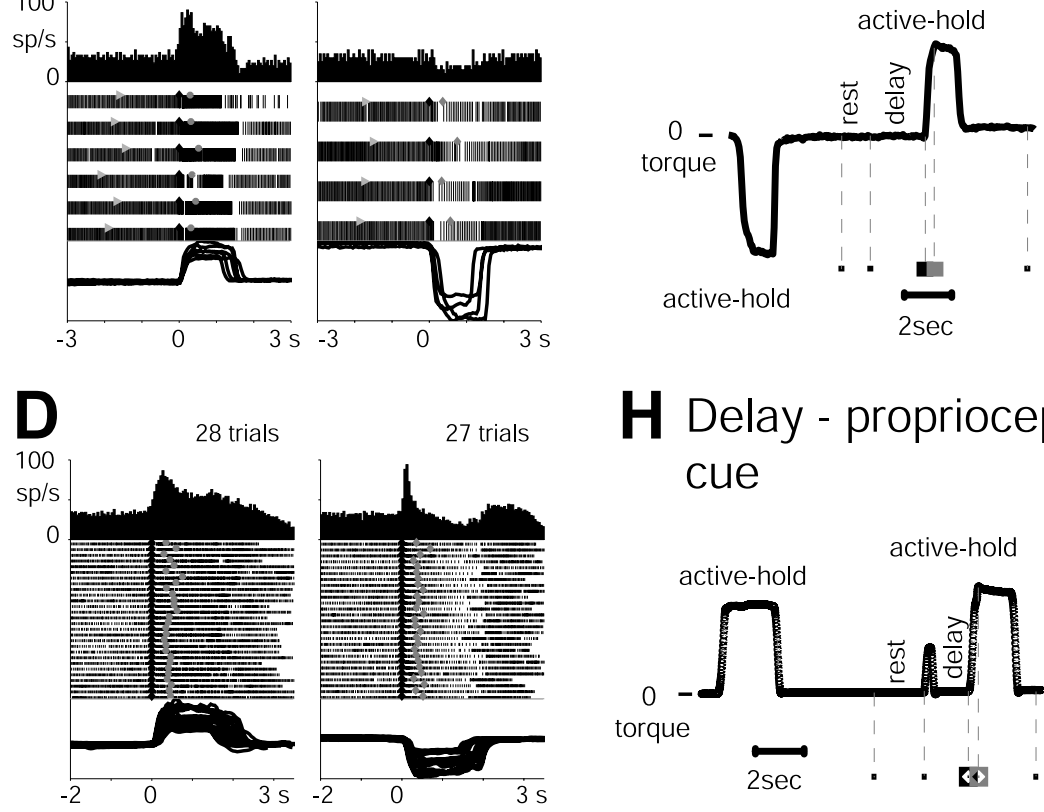

\section{H Delay - proprioceptive cue}

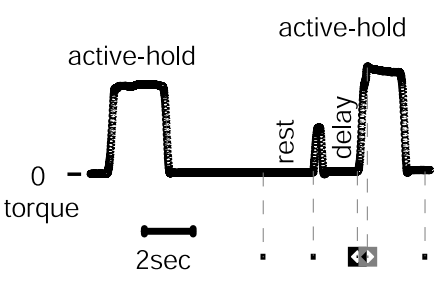

Figure 1. Behavioral tasks and unit responses for four spinal INs recorded during an alternating task $(A, E)$, a center-out task (B, $F)$, or a delayed response task with visual $(C, G)$ or proprioceptive $(D, H)$ cues. $A-D$, Histograms of unit firing are shown for flexion (left) and extension (right) trials, above raster plots and torque traces for individual trials. Histograms were computed using a 50 msec bin size without smoothing. $E-H$, Torque traces from two to four individual trials are shown for each task. The beginning and end of the dynamic torque, or active-ramp, period are shown by the black and gray symbols, respectively, on raster plots in $A-D$ and the torque traces in $E-H . G, H$, Small squares indicate, in order, the appearance of the center box, cue onset, and the disappearance of the center box (end of trial). Raster plots and torque traces are aligned on zero-crossing times of the torque trace in $A$ and on torque onset in $B-D$. Upward deflection of torque traces is flexion; downward deflection is extension (for all figures). 

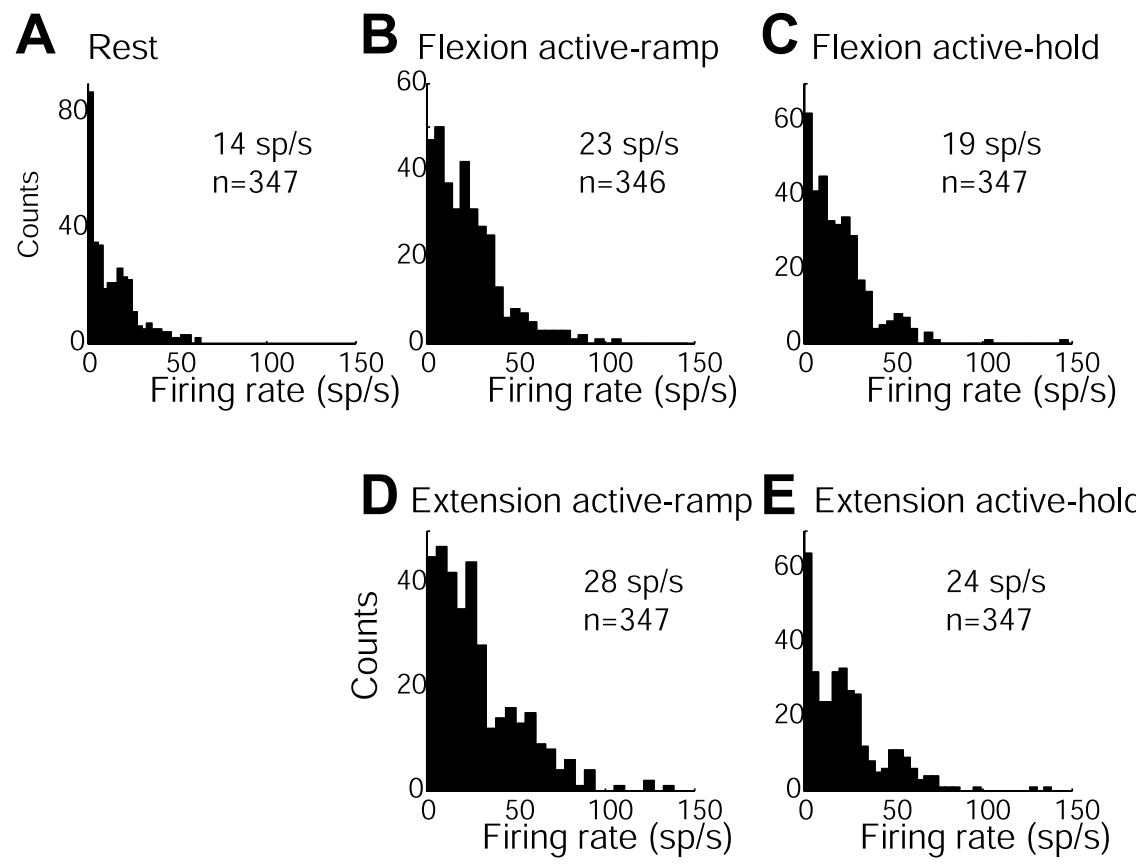

Figure 2. Distributions of average firing rates for single INs for the rest $(A)$, flexion active-ramp $(B)$, and active-hold $(C)$ and extension active-ramp $(D)$ and active-hold $(E)$ periods. The number of units and the mean rate are given for each distribution.

the time at which the histogram crossed the baseline mean +3 SD for excitatory responses and mean $-1.5 \mathrm{SD}$ for inhibitory responses (baseline period taken as 1000 to $500 \mathrm{msec}$ before torque onset). If the neuron had no baseline activity, the histogram was not smoothed, and the onset time was taken as the time of the first spike of the response.

\section{Results}

Firing rates

Most INs fired tonically during the rest period. On average, the population of INs tended to increase discharge rates for both flexion and extension torques (Fig. 1), although individual INs exhibited a variety of temporal response profiles [see companion paper (Prut and Perlmutter, 2003), Fig. 1; response profiles have been described in detail by Maier et al. (1998)]. The average rates of 374 well isolated (see Materials and Methods) INs were computed separately during rest, active-ramp, and active-hold periods. Data were analyzed only for neurons for which $\geq 500$ spikes were recorded (summed over all the epochs), which slightly biased our sample toward cells with high average firing rates. The population averages for mean firing rates in the active-hold periods in flexion and extension were 137 and 166\%, respectively, of the rate during the rest period (Fig. 2C,E). For the flexion and extension active-ramp periods, the average rates were 166 and $201 \%$ of the rest period rate, respectively (Fig. 2 B,D). The average increases in rate reflect the tendency of many INs to increase their discharge during both flexion and extension (Perlmutter et al., 1998).

The distribution of average firing rates for INs exhibited a nonmonotonic decay with a secondary peak at $\sim 50$ spikes (sp)/ sec during the active-hold period and at $\sim 30 \mathrm{sp} / \mathrm{sec}$ during the rest period (Fig. 2), suggesting the existence of IN subgroups. We examined the possibility of distinct groups related to the correlational linkages of the neurons with forearm muscles. U-INs had a significantly higher firing rate than $\mathrm{MNs}$ during rest, when $\mathrm{MNs}$ were silent, and active-hold periods. However, the average firing rates of cells with postspike or synchrony effects in STAs of fore- arm muscle EMG were not significantly different from those of U-INs ( $t$ test, $p>$ $0.05)$. This result is consistent with our earlier finding that there is no consistent relationship between the response profile of an IN and its correlational linkage with muscles (Maier et al., 1998). It appears that the magnitude and time course of modulated activity for INs with postspike and synchrony effects are similar.

\section{Regularity of IN firing}

Three groups of INs were defined by characteristic features in their autocorrelation functions. The majority of units exhibited a clear periodicity of firing throughout the task, although the periodicity varied during different behavioral epochs (Fig. $3 A, B)$. The second group of neurons was characterized by a random distribution of ISIs, similar to that of a Poisson process (Fig. 3C). The third group, observed least often, exhibited brief, high-frequency bursts of action potentials (Fig. 3D). Whereas the first two groups were not completely separable because their defining features represented extremes of a continuum, the third group appeared as a distinct, although small, subpopulation (3\% of all INs, consisting of seven strongly and four weakly bursting cells).

CV was used to quantify the tendency of INs to fire regularly. Sufficient data (see Materials and Methods) to calculate the average CV were obtained for 193 INs during the rest period and for 212 INs having a tonic response (i.e., steady firing rate) during the active-hold period. This difference in numbers resulted from epoch-related rate modulation and task variability (i.e., there was no rest period in monkey $\mathrm{B}$ ).

In general, INs fired regularly. Figure 4 shows the distribution of average CVs during the rest (Fig. $4 A$ ) and active-hold (Fig. $4 B$ ) periods. Almost all of the INs had an average CV of $<1$ (the CV of a Poisson process) during the rest and active-hold periods (Fig. $4 C$ ). Many INs (30 and 40\% of the population for rest and activehold periods, respectively) had an average $\mathrm{CV}$ of $<0.5$, a value indicating highly regular firing (Young et al., 1988) and approaching the CVs for MNs in our study and reported previously (0.2-0.3; Tanji and Kato, 1972; Sturm et al., 1997; Mattei and Schmied, 2002).

Simultaneous recordings of pairs of INs did not reveal any spatial organization of firing regularity. The extent to which INs exhibited periodic firing varied greatly from cell to cell, even for neurons that were located close together. The correlation coefficients between the average CVs of pairs of neurons recorded on the same electrode and recorded on different electrodes were not significantly different from each other or from zero $(p>0.05, t$ test on the $z$-transformed correlation coefficients). Bursting neurons, however, tended to be recorded at depths intermediate between those of the most dorsal and ventral neurons in a penetration, suggesting that they were located within the intermediate zone of the spinal gray matter.

\section{Relationships between firing regularity and behavior}

Average CV was inversely related to mean firing rate, for both rest and active-hold periods, when all data from the entire population 

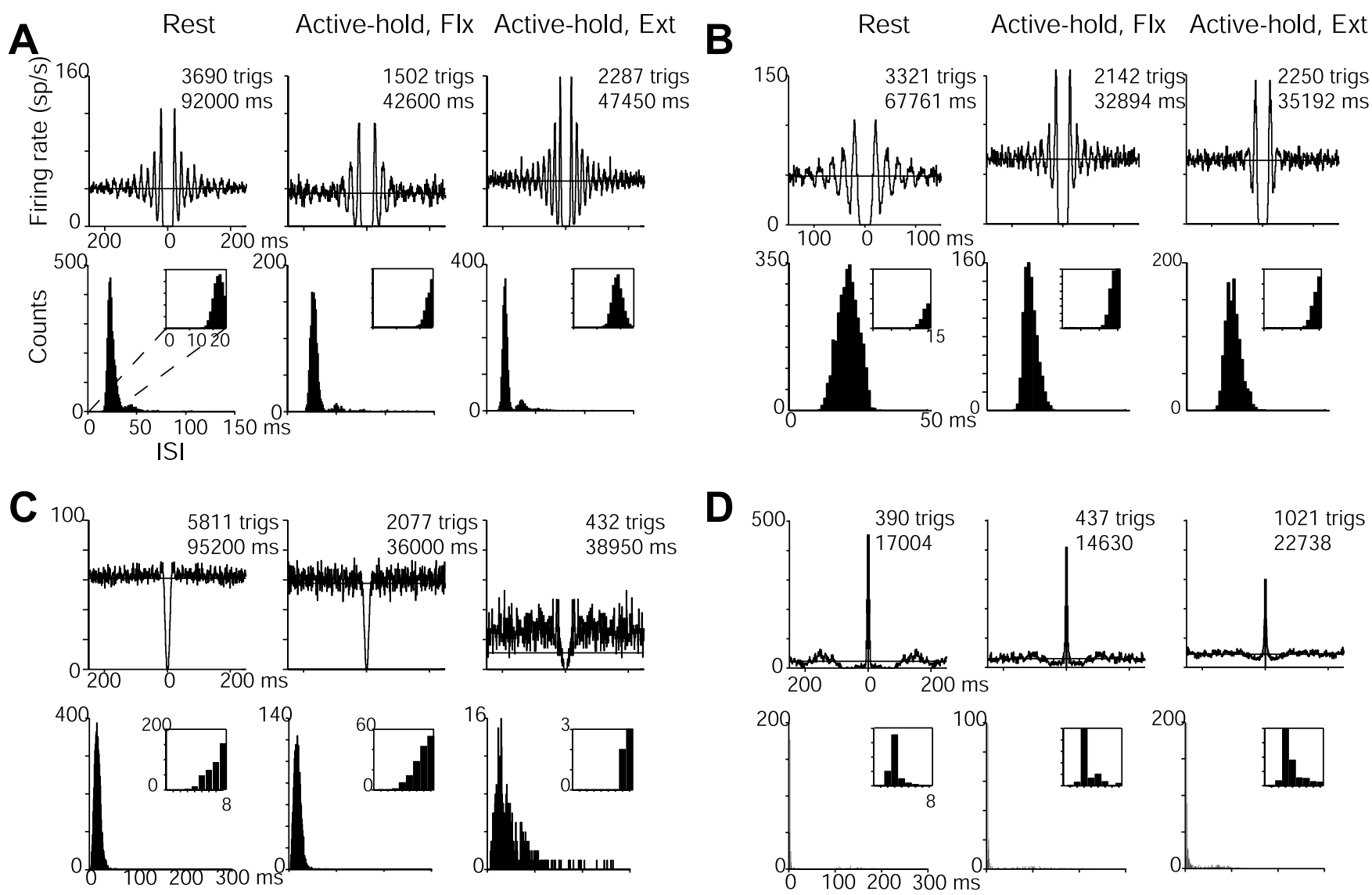

Figure 3. Autocorrelation functions (top) and ISI distributions (bottom) of four well isolated INs having clear refractory periods. Insets expand the near-zero segment of the ISI distribution. $A$, IN exhibiting regular firing, seen as periodicity in the autocorrelation function; the narrow ISI distribution shows regular firing at the single-spike level. B, IN exhibiting regular firing. C, IN exhibiting irregular firing, similar to a Poisson process. D, IN that fired in short, high-frequency bursts. Flx, Flexion; Ext, extension; trigs, trigger spikes.

of INs were combined (Fig. $4 A, B$ ). Many single INs exhibited this property as well (Fig. $3 A$, compare active-hold in flexion with rest or active-hold in extension). This relationship is characteristic of the response of neurons with constant refractory periods and afterhyperpolarizations (Goldberg et al., 1964; Piotrkiewicz, 1999; Powers and Binder, 2000). Some INs, however, did not exhibit this relationship between average CV and rate (Fig. 3B, compare active-hold in extension and rest).

To explore the factors affecting firing regularity, the $\mathrm{CV}_{\mathrm{i}}$ was examined as a function of mean firing rate and task epoch (Fig. 5). Many INs exhibited a monotonic decrease of $\mathrm{CV}_{i}$ with increasing rate across task epochs (Fig. 5A), whereas other cells exhibited more complex relationships. $\mathrm{CV}_{\mathrm{i}}$ increased with increasing rate across epochs for some INs (Fig. 5B,C), and for others, the $\mathrm{CV}_{\mathrm{i}}-$ rate relationship depended on the behavior. $\mathrm{CV}_{\mathrm{i}}-$ rate plots for the neurons in Figure $5 D-F$ were grouped into clusters by epoch (color data points), so that for a given level of firing rate, the regularity of each cell was quite variable. The epoch-related clusters often formed parallel clouds of points (Fig. $5 D-F)$. This suggested monotonic, often consistent, relationships between regularity and rate for a given behavior but steplike offsets in these relationships for different behaviors. The clusters for the neuron in Figure $5 D$ were not only parallel but also nonmonotonically arranged: $\mathrm{CV}_{\mathrm{i}}$ was highest during the activehold period in extension when firing rates were intermediate between those during the rest period (low rates) and the activehold period in flexion (high rates). Thus, for many neurons, there was a task-dependent impact on discharge regularity beyond the effect of any behaviorally related modulation of rate.
The heterogeneity of $\mathrm{CV}_{\mathrm{i}}$-rate dependencies made it difficult to define a robust parametric approach to quantify these relations. We estimated the contribution of the intrinsic properties of neurons (e.g., refractory period) by assuming that $\mathrm{CV}_{\mathrm{i}}$ was either a decreasing exponential or linear function of firing rate. Then we tested the residual values for epoch-related effects. Neurons were considered to have task-dependent $\mathrm{CV}_{\mathrm{i}}$-rate relationships only when the residuals from both exponential and linear fits (tested separately) had significant behavioral dependence (ANOVA, $p<$ 0.05 ). This test was performed for 188 single units with significant modulation of activity in one or both active-hold periods relative to the rest period or a significant preferred direction (flexion vs extension). Of these, 60 INs (32\%) had residuals that exhibited a significant relationship to behavior. For these cells, the relationship between $\mathrm{CV}_{\mathrm{i}}$ and firing rate was different for different phases of the task. Furthermore, an additional 26 (14\%) INs had a linear fit with a positive slope, indicating that their firing became more irregular with increasing rate. The results were essentially the same when this test was performed on data from only the last second of each epoch (instead of the entire epoch) to minimize effects of phasic rate modulation associated with torque onset.

In summary, changes in $\mathrm{CV}_{\mathrm{i}}$ across behavioral epochs could be accounted for by changes in mean firing rate for $54 \%$ of the INs. For the remaining $46 \%$ of the INs, the statistics of discharge during voluntary wrist movements did not appear to be determined solely by intrinsic properties. Instead, other behaviorally dependent factors, such as the discharge statistics of active inputs, contributed to discharge variability. 

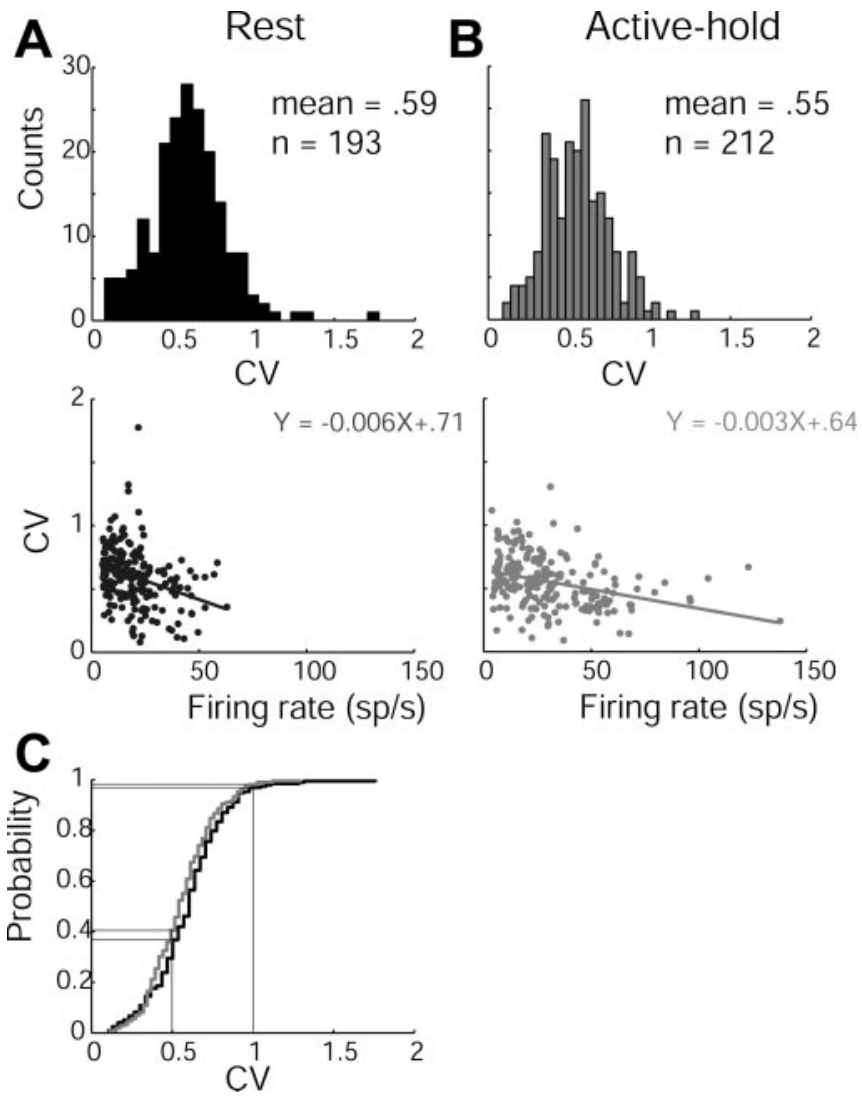

Figure 4. Distribution of average CVs (top) during rest period $(A)$ and the last 1 sec of the active-hold periods (flexion and extension combined) for cells exhibiting a tonic response ( $B$ ). Bottom graphs plot the relationship between average $\mathrm{CV}$ and average firing rate. Regression lines for the rest $(A)$ and active-hold $(B)$ periods have significant negative slopes $(p<0.01)$, indicating a tendency for increased regularity with increased rate, but were not significantly different from each other. The cumulative distributions of the average $\mathrm{CVs}_{\mathrm{s}}(\mathrm{C})$ for the rest (black line) and active-hold (gray line) periods show a tendency for average CVs to be lower during the active-hold period, which may be accounted for by the average increase in rate during this period.

\section{Firing properties of INs identified by correlational linkage} with muscles

Neurons with different correlational linkages with forearm muscles exhibited different distributions of CV during the generation of active torque (Fig. $6 \mathrm{~B}$ ). MNs were the most regular cells; the mean value of the average $\mathrm{CV}$ for $13 \mathrm{MNs}$ was $0.22 \pm 0.08 \mathrm{SD}$. INs with correlational linkages with muscles were the next most regular. Average CV was calculated for 79 INs with postspike effects, synchrony effects, or both in STAs of EMG recordings, with a mean of $0.48 \pm 0.17$ during the active-hold periods. INs with no observable effect on muscle activity were the most irregular cells. The mean average $\mathrm{CV}$ during the active-hold periods of $95 \mathrm{U}$-INs was $0.58 \pm 0.21$. The average CVs of U-INs and INs with correlational linkages with muscles were significantly different (Kruskal-Wallis test, $p<0.05$ ), although the two populations had similar firing rates in the active-hold periods. There was no significant difference between the average CVs of these populations during the rest period (Fig. 6A).

\section{Firing properties of INs identified by responses to sensory input}

Thirty-eight well isolated INs in monkey $\mathrm{H}$ had a clear response to passive displacement of the wrist. Three groups could be distinguished on the basis of their responses to the perturbations.
These groups exhibited different temporal firing properties and different firing patterns during generation of active torque.

A coactivation response (C-type; Fig. 7A) was observed in 11 cells $(29 \%)$. These INs fired short bursts of action potentials during the onset and usually the offset of perturbations in both flexion and extension. Often, the offset burst appeared weaker than the onset burst. A few cells fired only a single spike at the onset of the perturbation (in both directions), and these were considered to be C-type responses. C-type responses to passive displacements are consistent with input from fast-adapting cutaneous afferents (see Discussion).

A biphasic response (B-type; Fig. 7B) was observed in 19 cells $(50 \%)$. In its clearest form, the response consisted of an excitation for one direction of passive movement and an inhibition for the other direction. In these cases, there were responses of opposite polarity at the onset and offset of each perturbation (Fig. $7 B$ ). In some cases, the response during either perturbation onset or offset was weak. There were strong responses for the other phase of the displacement (offset or onset), which were of opposite polarity for flexion and extension. The B-type response is consistent with input from either muscle or joint receptors (see Discussion).

The third type of response was a monophasic response (Mtype; Fig. $7 C$ ), observed in eight (21\%) of the cells. This group of responses was more heterogeneous in appearance and properties compared with the previous two groups. The M-type responses usually occurred only at the onset of perturbations in one direction and were characterized by more sluggish temporal profiles.

The median onset time of the response to perturbation for all 38 INs was $25 \mathrm{msec}$ (mean, $29.2 \mathrm{msec}$ ). The average response latency of B-type cells was significantly shorter than that of C-type cells (means, 18 vs 45 msec; medians, 13 vs 35 msec; $t$ test, $p<0.01$ ). M-type cells exhibited intermediate response latencies.

The activity patterns of C- and B-type cells differed during active torque production (Figs. 7, 8) and reflected their responses to passive wrist displacement. C-type cells were characterized by low firing rates during static torque periods (both rest and activehold) and bidirectional increases in activity during torque onset and offset. B-type cells tended to have higher firing rates during the rest period than C-type cells and tonic activity during maintained torques. For the active-hold periods, 16 of 19 B-type cells had a preferred direction opposite to the displacement direction that elicited an excitatory response to perturbation. For 13 of these 16 neurons, the onset time of the response during active movement in the preferred direction was significantly earlier than for the response in the nonpreferred direction (means for all 16 neurons, $221 \pm 136$ vs $106 \pm 105$ msec before movement onset in the preferred and nonpreferred directions, respectively). During the active-ramp period, B-type neurons were either phasically excited bidirectionally or phasically excited for one direction of torque and phasically inhibited for the other direction.

Figure 9 shows the responses of two B-type neurons. The centrally driven and sensory response components can be seen during active movement in Figure 9B. There was an early increase in activity in the preferred direction (black), probably attributable to descending inputs. This was interrupted near torque onset by a phase of decreased firing, probably the inhibitory component of the B-type response to wrist displacement. In the nonpreferred direction (gray), the increase in activity began closer to torque onset, probably reflecting the excitatory component of the sensory response.

The temporal firing properties of B- and C-type INs also differed. C-type cells tended to be more irregular than B-type cells. 


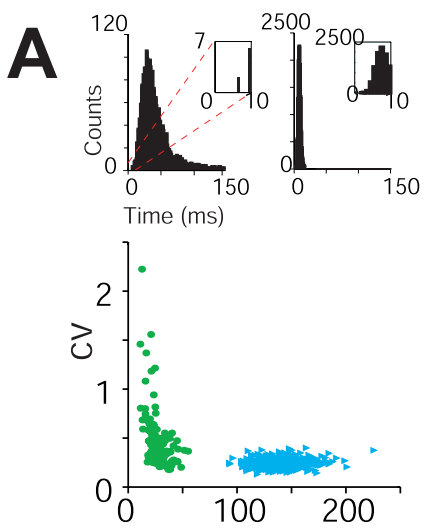

D
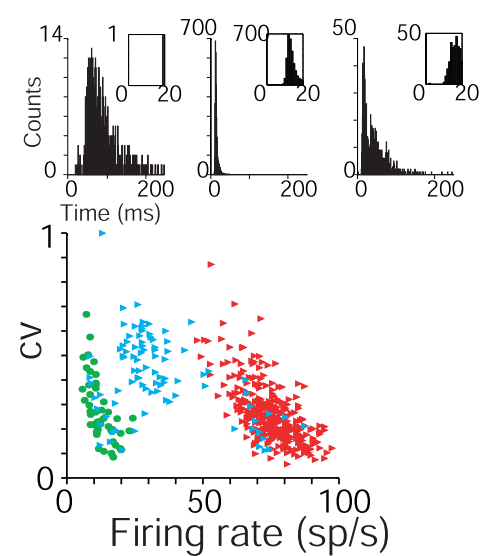
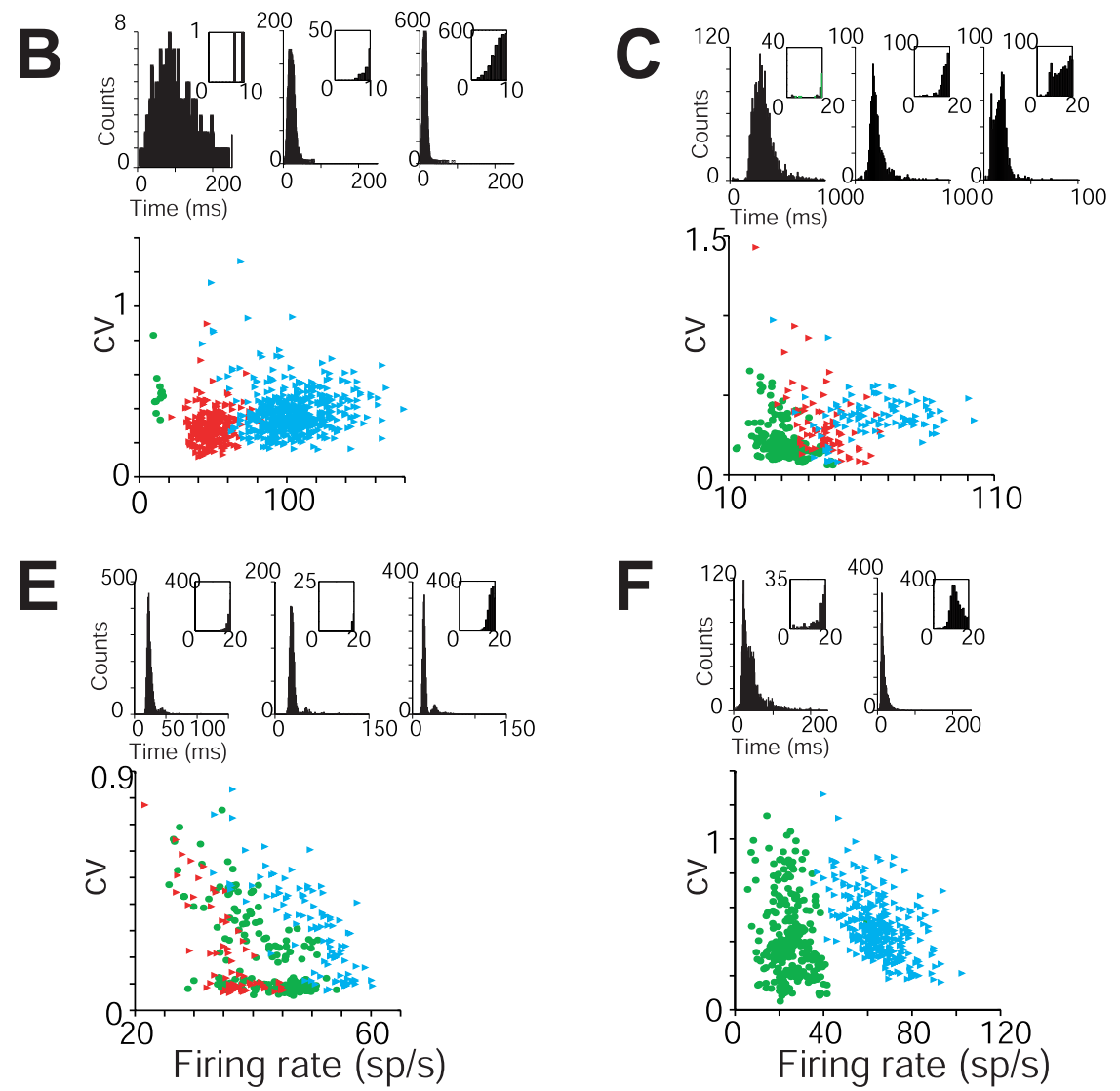

Figure 5. Relationships between $\mathrm{CV}_{\mathrm{i}}$ and firing rate for six well isolated INs $(A-F)$ during the rest period (green) and active-hold periods in flexion (red) and extension (blue). $C V_{i}$ and rate were computed from a fixed number of intervals ( 10 in $B-D, F, 20$ in $A, E$ ). ISI distributions for each epoch are shown above the plots; insets expand the near-zero segment of the distribution. The unit in A showed a monotonic decrease in $\mathrm{CV}_{\mathrm{i}}$ with increasing rate. Units in $B, C$, F exhibited an increase in $C \mathrm{~V}_{\mathrm{i}}$ with increasing rate. For units in $D, E, C \mathrm{~V}_{\mathrm{i}}-$ rate plots for different task epochs had similar slopes but different $y$-intercepts.

Average CVs were computed for each of the 38 INs in five different epochs: rest period and flexion and extension activeramp and active-hold periods (Fig. 10). The two distributions were significantly different (Kolmogorov-Smirnov, $p<$ 0.001 ), although this difference can be accounted for by the lower mean firing rates of C-type cells.

Finally, the behavioral dependence of discharge regularity was significantly different for B- and C-type cells. The $\mathrm{CV}_{\mathrm{i}}-$ rate data were fit with exponential and linear functions as described previously. There was a significant effect of behavior on the residuals of the fits for 11 of 19 (58\%) of the B-type cells, zero of five ( $0 \%)$ of the C-type cells (there were not enough data for the other six C-type cells to perform this analysis), and one of eight (13\%) of the M-type cells. In addition, two other B-type cells had a linear $\mathrm{CV}_{\mathrm{i}}$-rate relationship with a positive slope. All B-type cells with epoch-related $\mathrm{CV}_{\mathrm{i}}$-rate relationships had features similar to those shown in Figure 11. During the active-hold period in the preferred direction (flexion), the neuron discharged less regularly than expected for a monotonic relationship between $\mathrm{CV}_{\mathrm{i}}$ and rate.

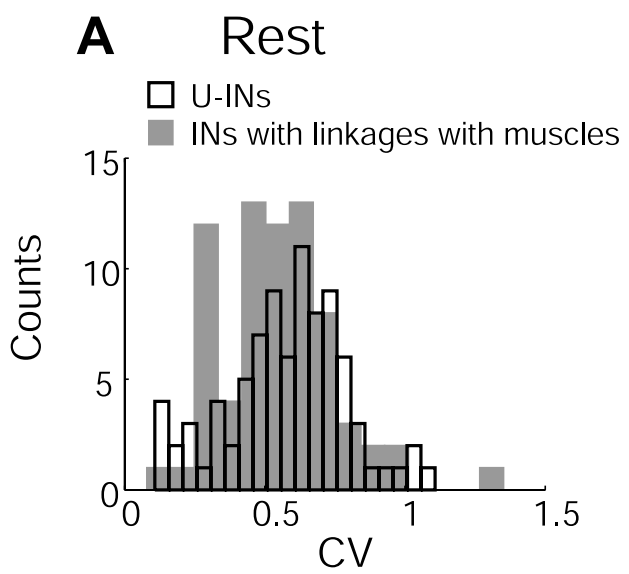

\section{B Active-hold}

Figure 6. Distribution of average CVs during the rest period $(A)$ and the active-hold periods ( $B$; flexion and extension combined) for U-INs (white bars) and INs with correlational linkages with muscles (gray bars). The mean average CV of U-INs during the rest period $(0.58 ; n=86)$ was not significantly different from the mean $(0.56 ; n=73)$ for INs with correlational linkages with muscles (Kruskal-Wallis, $p>0.14)$. In contrast, the mean average CVs during the active-hold periods of U-INs $(0.58 ; n=95)$ and INs with correlational linkages with muscles $(0.48 ; n=79)$ were significantly different (Kruskal-Wallis, $p<0.0002)$. The arrow in $B$ shows the mean average $C V$ of 13 MNs during the active-hold period in their preferred direction.

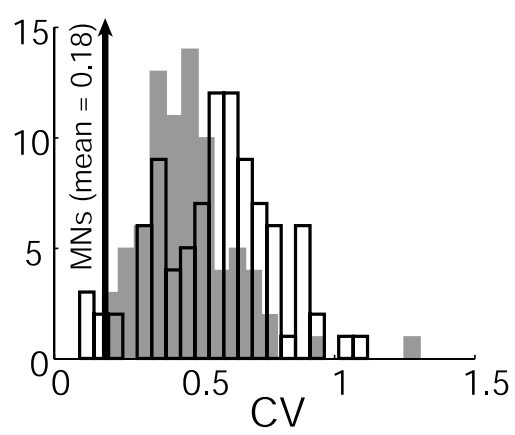

\section{Discussion}

Spinal versus cortical activity

Many INs were active when no torque was exerted at the wrist and during active torque production in both flexion and extension, as reported previously (Maier et al., 1998). This contrasts with the behavior of premotor cells in primary motor cortex, which are 

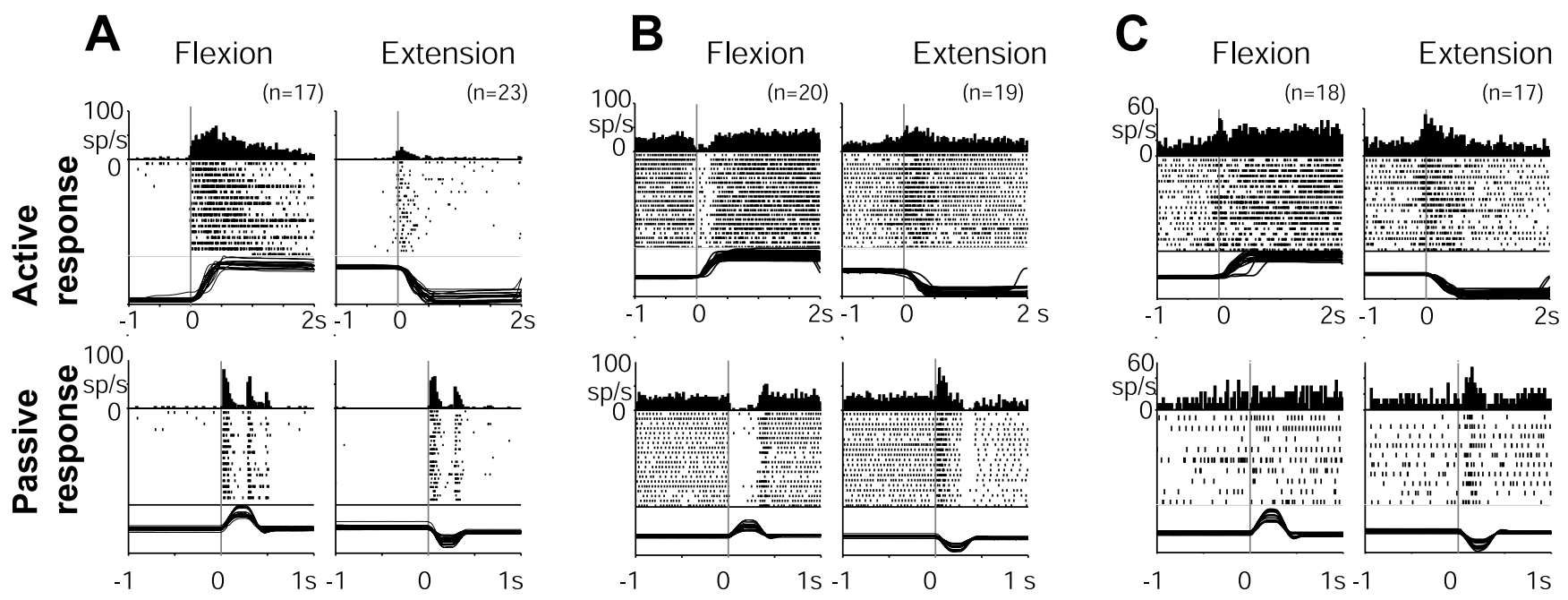

Figure 7. Activity of three INs $(A-C)$ during active generation of torque (top) and responses to passive wrist displacement (bottom). Histograms of unit firing are shown for flexion (left) and extension (right) above raster plots and torque traces for individual trials. Active responses are aligned on torque onset, and passive responses are aligned on perturbation onset. $B$, IN with a coactivation (C-type) response. B, IN with a biphasic (B-type) response. C, IN with a monophasic (M-type) response. M-type responses were not as stereotypical in appearance as B- and C-type responses.

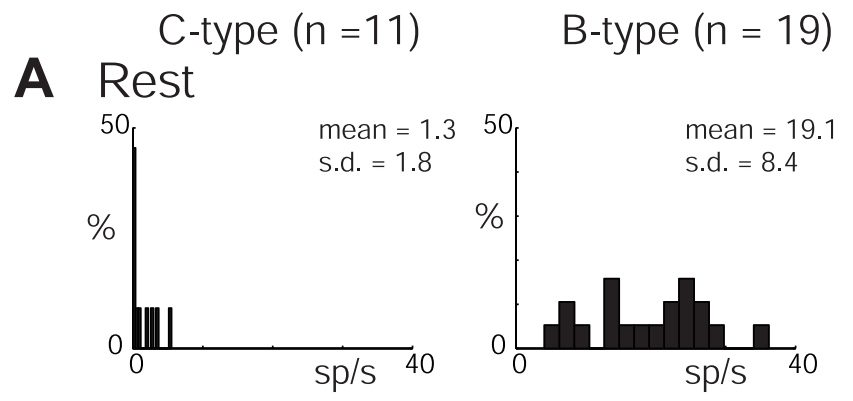

B Active-ramp
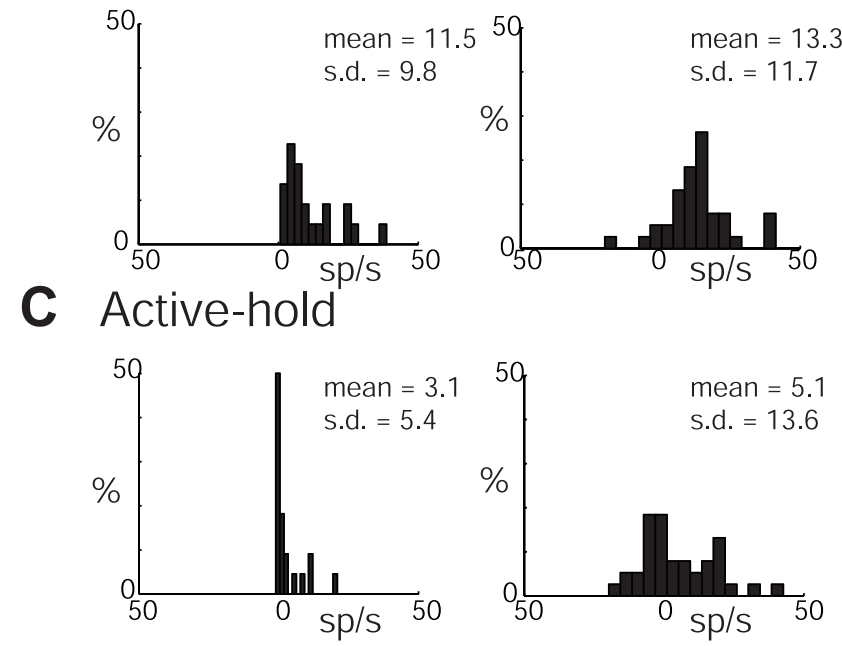

Figure 8. Rate distributions of C-type (left) and B-type (right) INs during rest $(A)$, activeramp $(B)$, and active-hold ( $C$ periods (flexion and extension combined). $A$, Absolute firing rate. $B, C$, Rate modulation relative to rest. Mean and $S D$ are given for each plot.

mostly silent during rest and are active unidirectionally (Cheney and Fetz, 1980). These properties suggest that spinal circuitry, unlike cortical premotor neurons, is constantly in a "ready" mode that can rapidly translate inputs into muscle activity.

A second difference between the firing pattern of spinal and cortical neurons is the tendency for INs to fire more regularly, with CVs of $<1$. Cerebral cortical neurons have reported CVs of
$>1$ (Softky and Koch, 1993; Lee et al., 1998; Stevens and Zador, 1998). It bears noting that our CVs were calculated during a relatively constant behavioral state (static torque generation), whereas much of the cortical data on variability were recorded from visual areas as monkeys viewed dynamic visual stimuli. The impact of behavioral context on our conclusions awaits an analysis of motor cortical regularity during the wrist flexion-extension task.

Lumbar INs of neonatal rats are capable of precise and reproducible firing in vitro (Beierholm et al., 2001), as are cortical neurons (Mainen and Sejnowski, 1995), suggesting that they can reliably follow high-frequency synaptic potentials. In neonatal rats, single neurons may respond very accurately and consistently to synaptic inputs, although their firing during individual cycles of pharmacologically induced locomotion appears to be variable (Tresch and Kiehn, 2000). Our results, however, suggest that cervical INs do not operate in this regime when maintained torques are exerted at the wrist. INs express very regular firing, even in single trials, implying that the current arriving at the soma is relatively constant. Under such conditions, information is carried primarily by the mean firing rate, and the ability of the cell to follow rapid changes of input may be limited (Abeles and Lass, 1975).

\section{Modulation of $\mathrm{CV}$ as a function of firing rate and behavioral mode}

Most neurons respond to increasing amounts of injected current with increasing rates of discharge and decreasing variability of interspike intervals (Mainen and Sejnowski, 1995; Stevens and Zador, 1998; Powers and Binder, 2000). This monotonic relationship between mean rate and CV can be demonstrated mathematically in models of neuron firing that include a refractory period (Goldberg et al., 1964). At higher firing rates, the refractory period becomes a larger percentage of the interspike interval, stabilizing its length. In our study, however, $46 \%$ of the INs did not exhibit this property, suggesting that the intrinsic properties of the cells did not account entirely for their firing regularity. For many of these cells, the CV-rate relationship depended on the monkey's behavior.

Theoretical studies (Segundo et al., 1968; Salinas and Sejnowski, 2000; Svirskis and Rinzel, 2000) have reasoned that un- 


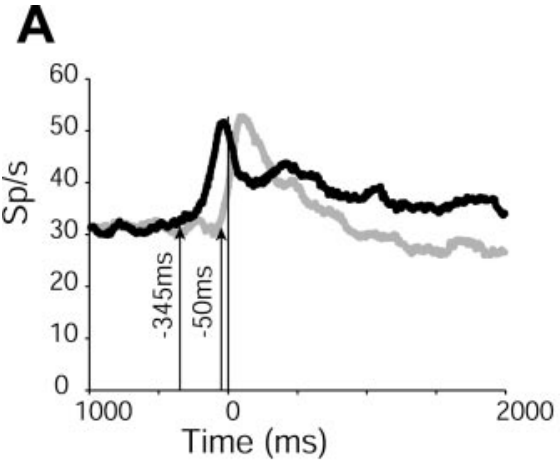

B

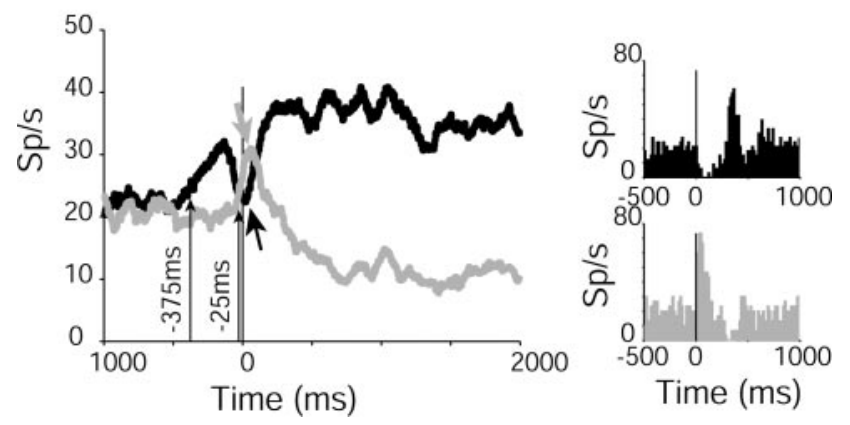

Figure 9. Histograms of firing during active generation of torque (left) and passive wrist displacement (right) for two INs $(A, B)$ with B-type responses to wrist perturbations. Black histograms show activity for the direction of movement that produced the largest response during the active-hold period (i.e., preferred direction for active movements). Gray histograms show activity for the opposite direction. Histograms are aligned on movement onset (left) or stimulus onset (right). Both INs have opposite preferred directions for active and passive movements. Small arrowheads indicate onset times for active movement. Both neurons had earlier onset times for active movements in the preferred than the nonpreferred direction. In $B$, phasic increase (large gray arrow) and decrease (large black arrow) in firing near movement onset probably reflect the sensory response of the neuron to wrist displacement (note directional and temporal similarity with passive responses shown to the right).

der specific circumstances, the firing statistics of a neuron will reflect the statistics of the inputs of the cell. This occurs when a few strong synaptic inputs dominate the membrane potential of the cell or when the cell receives inputs that are homogeneously weak but correlated, even slightly. If its inputs are homogeneously weak and uncorrelated, the cell fires regularly, regardless of the temporal structure of the inputs. Such modeling has led to the postulate that correlated input is the source of the discharge variability exhibited by cortical neurons (Stevens and Zador, 1998).

In our study, different descending and sensory pathways are active during the rest and active-hold periods and could account for nonmonotonically decreasing CV-rate relationships. Many INs became more irregular as their firing rate increased during active-hold periods. This is consistent with stronger input from motor cortex because corticospinal neurons increase their firing rate during active torques (Cheney and Fetz, 1980), and the discharge of motor cortical neurons tends to be irregular and somewhat correlated (Lee et al., 1998). $\mathrm{CV}_{\mathrm{i}}$-rate plots that were parallel but offset (e.g., Fig. 5D-F) suggest that descending inputs during flexion and extension had different temporal statistics.

Alternatively, changes in firing characteristics could reflect modulatory effects on the intrinsic properties of the neurons. Monoamines induce plateau potentials in MNs (Hounsgaard et al., 1988; White et al., 1996; Heckman and Lee, 1999) and alter the firing pattern of INs (Jankowska et al., 2000) and MNs (Rekling et al., 2000). Such effects could influence the discharge regularity of neurons as they do their input-output transformation. Jacobs et al. (2002) has postulated that serotonin-induced changes in spinal neuron excitability have significant functional consequences: facilitation of motor output and suppression of sensory inflow during movement.

Data from INs with B-type responses to passive displacements of the hand support the hypothesis that shifts in temporal properties reflect activation of inputs with different firing statistics. These neurons were excited by perturbations in a direction opposite to the preferred direction for active movement, suggesting a consistent involvement in the recruitment of agonist muscles for both voluntary and reflex movements. Consequently, the increased discharge during the active-hold period in the preferred direction was likely of central, not peripheral, origin. This inference is supported by the onset times of responses in the preferred direction of most B-type cells: before movement onset and significantly earlier than responses in the nonpreferred direction. These neurons exhibited higher CVs for a given mean firing rate during the active-hold period in the preferred direction than the nonpreferred direction. Together, these data suggest that descending inputs for the preferred direction induced irregular firing. During movements in the nonpreferred direction, peripheral feedback from, for example, regularly firing muscle afferents (Matthews and Stein, 1969) contributed to more regular discharge.

This type of analysis may help us identify the inputs that drive IN discharge during particular behaviors. It will require, however, characterization of the temporal properties of descending and peripheral inputs and the extent to which they contribute to the statistics of IN firing.

The current study does not address whether behaviorally dependent temporal properties are important mechanisms for generating muscle activity. Signals from different sources with similar mean firing rates could be translated differently into postsynaptic firing attributable to different statistics (Baker et al., 2001), or INs may filter high-frequency inputs to the cord because neurons that fire regularly cannot follow fast temporal fluctuation of their inputs (Abeles and Lass, 1975). Whether these factors are at work in spinal processing of premotor signals awaits a direct investigation.

\section{Input-to-output mapping in spinal circuitry}

The response properties of INs during active movement do not appear to be related to their linkages to muscles (Perlmutter et al., 1998). It is possible that neurons that we identified as U-INs did have correlational linkages to unrecorded motor units. Our EMG electrodes did not sample all motor units within an implanted muscle, nor did we record from all muscles of the arm. However, the diversity of responses of INs with postspike or synchrony effects suggests that responses and muscle linkages are not strongly related.

The present results indicate that the firing properties of INs are correlated with their responses to mechanical stimuli and, therefore, with the somatosensory inputs they receive. The responses of C- and B-type neurons to passive displacements and active torque generation are consistent with input from fastadapting cutaneous afferents (Vallbo and Johansson, 1984; Edin and Abbs, 1991) and muscle or joint receptors (Matthews, 1981), respectively. Similar responses to passive joint rotation of postcentral cortical neurons with identified cutaneous or muscle afferent input (Soso and Fetz, 1980) support this classification. Cells with B- and C-type sensory responses had different activity 


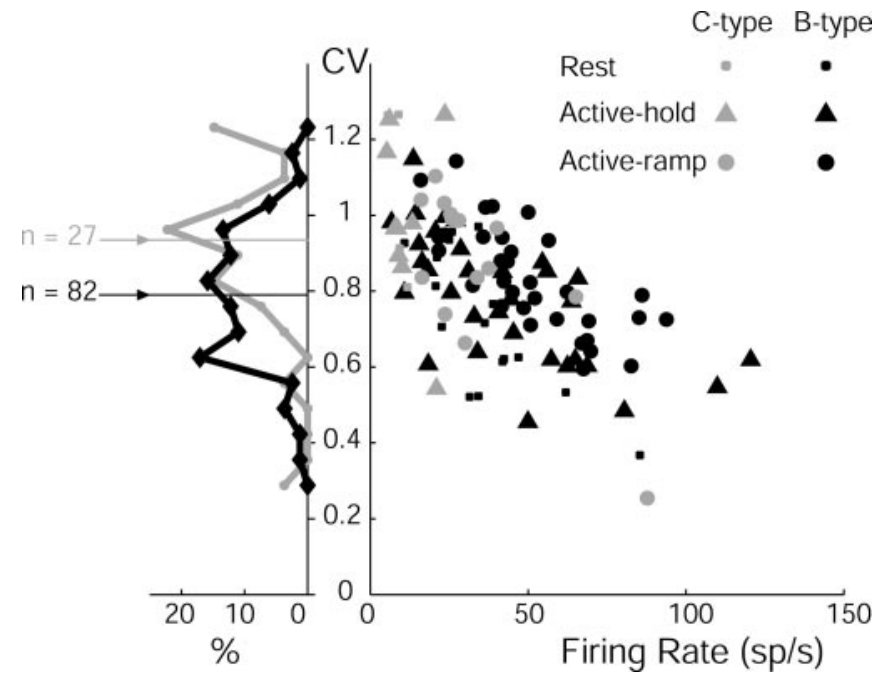

Figure 10. Distributions of average CVs for C-type (gray) and B-type (black) INs (left) and the relationship between average $\mathrm{CV}$ and firing rate during rest (small circles), active-ramp (large circles), and active hold (triangles) periods.

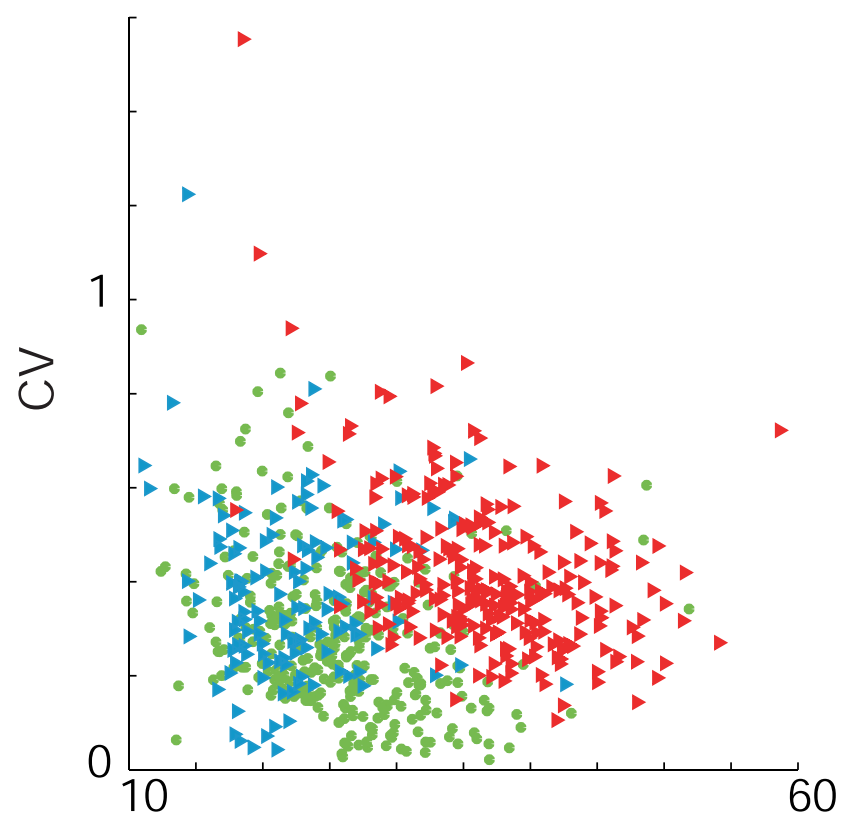

Firing rate $(\mathrm{sp} / \mathrm{s})$

Figure 11. Relationship between $\mathrm{CV}_{\mathrm{i}}$ and firing rate for a B-type cell with a significant behavioral effect on the residuals. Data are plotted for rest period (green) and active-hold periods in flexion (red) and extension (blue).

patterns and temporal firing characteristics during active movement, indicating that they contribute differently to the generation of torque.

This result is not predicted from findings on the distribution of afferent axons within the spinal gray matter. INs of the same functional group randomly sample the large number of peripheral inputs entering the cord (Harrison and Jankowska, 1985; Davies and Edgley, 1994). Consequently, we expected INs to exhibit a larger spectrum of responses to somatosensory stimuli. More varied sensory convergence might be revealed by further examination of B- and C-type responses or by applying a wider set of sensory stimuli rather than only flexion and extension perturbations of the wrist.
Correlation between response properties and sensory inputs but not output linkages would suggest that a network lacks rigid input-output mappings. This would be consistent with the view of the spinal cord as a site of convergence and integration of descending and peripheral signals. A given input is processed through multiple pathways that combine it with other information in a behaviorally dependent manner, providing flexibility to the premotor network.

Relationships were found between the regularity of discharge and the connectivity of spinal neurons: MNs fired the most regularly; cells without correlational linkages with muscles fired the most irregularly; and INs with correlational linkages with muscles had CVs that were intermediate. If irregular firing of INs reflects correlated inputs, this result suggests that information is decorrelated as it is transferred through the spinal network. A more explicit study of correlated firing between INs is the focus of the companion paper (Prut and Perlmutter, 2003).

\section{References}

Abeles M, Lass Y (1975) Transmission of information by the axon. II. The channel capacity. Biol Cybern 19:121-125.

Baker SN, Spinks R, Jackson A, Lemon RN (2001) Synchronization in monkey motor cortex during a precision grip task. I. Task-dependent modulation in single-unit synchrony. J Neurophysiol 85:869-885.

Beierholm U, Nielsen CD, Ryge J, Alstrom P, Kiehn O (2001) Characterization of reliability of spike timing in spinal interneurons during oscillating inputs. J Neurophysiol 86:1858-1868.

Bennett BD, Wilson CJ (1999) Spontaneous activity of neostriatal cholinergic interneurons in vitro. J Neurosci 19:5586-5596.

Butt SJ, Harris-Warrick RM, Kiehn O (2002) Firing properties of identified interneuron populations in the mammalian hindlimb central pattern generator. J Neurosci 22:9961-9971.

Calvin WH, Stevens CF (1968) Synaptic noise and other sources of randomness in motoneuron interspike intervals. J Neurophysiol 31:574-587.

Cheney PD, Fetz EE (1980) Functional classes of primate corticomotoneuronal cells and their relation to active force. J Neurophysiol 44:773-791.

Davies HE, Edgley SA (1994) Inputs to group II-activated midlumbar interneurones from descending motor pathways in the cat. J Physiol (Lond) 479:463-473.

Dengler R, Wolf W, Schubert M, Struppler A (1986) Discharge pattern of single motor units in basal ganglia disorders. Neurology 36:1061-1066.

Edin BB, Abbs JH (1991) Finger movement responses of cutaneous mechanoreceptors in the dorsal skin of the human hand. J Neurophysiol 65:657-670.

Floeter MK, Sholomenko GN, Gossard JP, Burke RE (1993) Disynaptic excitation from the medial longitudinal fasciculus to lumbosacral motoneurons: modulation by repetitive activation, descending pathways, and locomotion. Exp Brain Res 92:407-419.

Goldberg JM, Adrian HO, Smith FD (1964) Response of neurons of the superior olivary complex of the cat. J Neurophysiol 27:706-749.

Gonzalez-Forero D, De La Cruz RR, Delgado-Garcia JM, Pastor AM (2001) Reversible deafferentation of abducens motoneurons and internuclear neurons with tetanus neurotoxin. NeuroReport 12:753-756.

Harrison PJ, Jankowska E (1985) Organization of input to the interneurones mediating group I non-reciprocal inhibition of motoneurones in the cat. J Physiol (Lond) 361:403-418.

Heckman CJ, Lee RH (1999) Synaptic integration in bistable motoneurons. Prog Brain Res 123:49-56.

Hounsgaard J, Hultborn H, Jespersen B, Kiehn O (1988) Bistability of alpha-motoneurones in the decerebrate cat and in the acute spinal cat after intravenous 5-hydroxytryptophan. J Physiol (Lond) 405:345-367.

Jacobs BL, Martin-Cora FJ, Fornal CA (2002) Activity of medullary serotonergic neurons in freely moving animals. Brain Res Brain Res Rev 40:4552.

Jankowska E, Hammar I, Chojnicka B, Heden CH (2000) Effects of monoamines on interneurons in four spinal reflex pathways from group I and/or group II muscle afferents. Eur J Neurosci 12:701-714.

LaBella LA, Niechaj A, Rossignol S (1992) Low-threshold, short-latency cutaneous reflexes during fictive locomotion in the "semi-chronic" spinal cat. Exp Brain Res 91:236-248. 
Lee D, Port NL, Kruse W, Georgopoulos AP (1998) Variability and correlated noise in the discharge of neurons in motor and parietal areas of the primate cortex. J Neurosci 18:1161-1170.

Maier MA, Perlmutter SI, Fetz EE (1998) Response patterns and force relations of monkey spinal interneurons during active wrist movement. J Neurophysiol 80:2495-2513.

Mainen ZF, Sejnowski TJ (1995) Reliability of spike timing in neocortical neurons. Science 268:1503-1506.

Mattei B, Schmied A (2002) Delayed and prolonged effects of a near threshold EPSP on the firing time of human alpha-motoneurones. J Physiol (Lond) 538:849-865.

Matthews PB (1981) Muscle spindles: their message and their fusimotor supply. In: Handbook of physiology (Brooks VB, ed), pp 189-228. Bethesda, MD: American Physiological Society.

Matthews PB, Stein RB (1969) The regularity of primary and secondary muscle spindle afferent discharges. J Physiol (Lond) 202:59-82.

Pearson KG (1995) Proprioceptive regulation of locomotion. Curr Opin Neurobiol 5:786-791.

Perlmutter SI, Maier MA, Fetz EE (1998) Activity of spinal interneurons and their effects on forearm muscles during voluntary wrist movements in the monkey. J Neurophysiol 80:2475-2494.

Person RS, Kudina LP (1972) Discharge frequency and discharge pattern of human motor units during voluntary contraction of muscle. Electroencephalogr Clin Neurophysiol 32:471-483.

Piotrkiewicz M (1999) An influence of afterhyperpolarization on the pattern of motoneuronal rhythmic activity. J Physiol (Paris) 93:125-133.

Powers RK, Binder MD (2000) Relationship between the time course of the afterhyperpolarization and discharge variability in cat spinal motoneurones. J Physiol (Lond) 528:131-150.

Prut Y, Fetz EE (1999) Primate spinal interneurons show pre-movement instructed delay activity. Nature 401:590-594.

Prut Y, Perlmutter SI (2003) Firing properties of spinal interneurons during voluntary movement. II. Interactions between spinal neurons. J Neurosci 23:9611-9619.

Rees A, Sarbaz A, Malmierca MS, Le Beau FE (1997) Regularity of firing of neurons in the inferior colliculus. J Neurophysiol 77:2945-2965.

Rekling JC, Funk GD, Bayliss DA, Dong XW, Feldman JL (2000) Synaptic control of motoneuronal excitability. Physiol Rev 80:767-852.

Riehle A, Grun S, Diesmann M, Aertsen A (1997) Spike synchronization and rate modulation differentially involved in motor cortical function. Science 278:1950-1953.

Salinas E, Sejnowski TJ (2000) Impact of correlated synaptic input on out- put firing rate and variability in simple neuronal models. J Neurosci 20:6193-6209.

Segundo JP, Moore GP, Stensaas L, Bullock TH (1963) Sensitivity of neurons in Aplysia to temporal patterns of arriving impulses. J Exp Biol 40:643-667.

Segundo JP, Perkel DH, Wyman H, Hegstad H, Moore GP (1968) Inputoutput relations in computer-simulated nerve cells. Influence of the statistical properties, strength, number and inter-dependence of excitatory pre-synaptic terminals. Kybernetik 4:157-171.

Softky WR, Koch C (1993) The highly irregular firing of cortical cells is inconsistent with temporal integration of random EPSPs. J Neurosci 13:334-350.

Soso MJ, Fetz EE (1980) Responses of identified cells in postcentral cortex of awake monkeys during comparable active and passive joint movements. J Neurophysiol 43:1090-1110.

Stein RB, Capaday C (1988) The modulation of human reflexes during functional motor tasks. Trends Neurosci 11:328-332.

Stevens CF, Zador AM (1998) Input synchrony and the irregular firing of cortical neurons. Nat Neurosci 1:210-217.

Sturm H, Schmied A, Vedel JP, Pagni S (1997) Firing pattern of typeidentified wrist extensor motor units during wrist extension and hand clenching in humans. J Physiol (Lond) 504:735-745.

Svirskis G, Rinzel J (2000) Influence of temporal correlation of synaptic input on the rate and variability of firing in neurons. Biophys 79:629-637.

Tanji J, Kato M (1972) Discharges of single motor units at voluntary contraction of abductor digiti minimi muscle in man. Brain Res 45:590-593.

Tresch MC, Kiehn O (2000) Population reconstruction of the locomotor cycle from interneuron activity in the mammalian spinal cord. J Neurophysiol 83:1972-1978.

Vallbo AB, Johansson RS (1984) Properties of cutaneous mechanoreceptors in the human hand related to touch sensation. Hum Neurobiol 3:3-14.

White SR, Fung SJ, Jackson DA, Imel KM (1996) Serotonin, norepinephrine and associated neuropeptides: effects on somatic motoneuron excitability. Prog Brain Res 107:183-199.

Wiegner AW, Wierzbicka MM, Davies L, Young RR (1993) Discharge properties of single motor units in patients with spinal cord injuries. Muscle Nerve 16:661-671.

Young ED, Robert JM, Shofner WP (1988) Regularity and latency of units in ventral cochlear nucleus: implications for unit classification and generation of response properties. J Neurophysiol 60:1-29. 